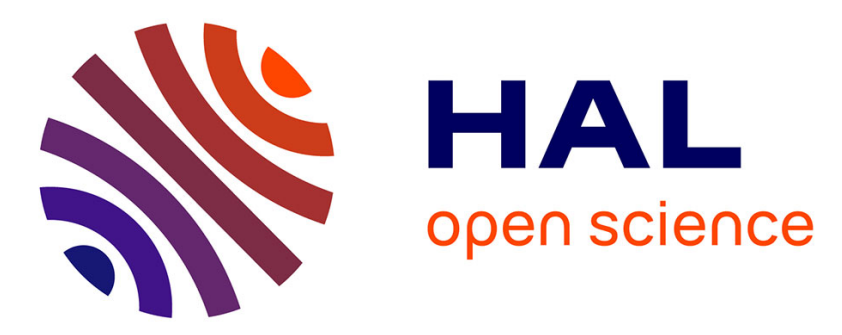

\title{
Recognition of Linear-Slender Context-Free Languages by Real Time One-Way Cellular Automata
}

\author{
Véronique Terrier
}

\section{To cite this version:}

Véronique Terrier. Recognition of Linear-Slender Context-Free Languages by Real Time One-Way Cellular Automata. 21st Workshop on Cellular Automata and Discrete Complex Systems (AUTOMATA), Jun 2015, Turku, Finland. pp.251-262, 10.1007/978-3-662-47221-7_19 . hal-01442477

\section{HAL Id: hal-01442477 \\ https://hal.inria.fr/hal-01442477}

Submitted on 20 Jan 2017

HAL is a multi-disciplinary open access archive for the deposit and dissemination of scientific research documents, whether they are published or not. The documents may come from teaching and research institutions in France or abroad, or from public or private research centers.
L'archive ouverte pluridisciplinaire HAL, est destinée au dépôt et à la diffusion de documents scientifiques de niveau recherche, publiés ou non, émanant des établissements d'enseignement et de recherche français ou étrangers, des laboratoires publics ou privés. 


\title{
Recognition of linear-slender context-free languages by real time one-way cellular automata
}

\author{
Véronique Terrier \\ GREYC, UMR 6072, Université de Caen Basse-Normandie \\ Campus Côte de Nacre, boulevard Maréchal Juin, 14032 Caen, France \\ veronique.terrier@unicaen.fr
}

\begin{abstract}
A linear-slender context-free language is a context-free language whose number of words of length $n$ is linear in $n$. Its structure has been finely characterized in a work of Ilie, Rozenberg and Salomaa. Thanks to this characterization, we show that every linear-slender context-free language is recognizable by a real time one-way cellular automaton.
\end{abstract}

\section{Introduction}

One-way cellular automaton (OCA) is one of the simplest parallel recognizing devices. Since its introduction by Dyer [5], it has been the subject of much interest. Numerous studies have been directed towards the real time OCA, the class of languages decidable in minimal time, and have already provided a good understanding of its abilities and limitations.

In particular, it is known that the real time OCA and the family of context free languages are incomparable [12]. This calls into question which languages they have in common. That is not the case for deterministic CFL: there exits a LL(1) CFL which is not a real time OCA one [11]. On the other hand, linear CFL and visible pushdown languages have been proved to be real time OCA ones $[3,11]$.

With the aim to identify more common languages, we will take into consideration the counting function that measures the number of words of length $n$ in the language. Noticing that all CFL known to be not real time OCA have their counting function of exponential order, the best is to look at sparse languages. These are the poly-slender CFL whose counting functions are polynomial in $n$ and, more specifically, the linear-slender CFL whose counting functions are linear. The purpose here is to show that linear-slender CFL are real time OCA ones.

The present work essentially relies on a paper by Ilie, Rozenberg and Salomaa which presents a characterization of poly-slender CFL in terms of Dyck loops [7]. Culík's OCA which recognizes in real time the language $\left\{a^{n} b^{n+m} a^{m}: n, m \geq 0\right\}$ and Okhotin's characterization of real time OCA by linear conjunctive grammars will come also into play $[2,10]$. 


\section{Preliminary}

\subsection{Basic notions}

We first recall some basic definitions and notations.

For any language $L$, the number of words in $L$ of length $n$ is denoted by $\sharp_{n}(L)$. For an integer $k$, a language $L$ is $k$-poly-slender if $\sharp_{n}(L)$ is in $\mathcal{O}\left(n^{k}\right)$.

A language $L$ is poly-slender if $L$ is $k$-poly-slender for some $k$. A language $L$ is linear-slender if $\sharp_{n}(L)$ is in $\mathcal{O}(n)$.

A language $L$ is bounded if there exists a finite number of words $u_{1}, u_{2}, \cdots, u_{k}$ such that $L \subseteq u_{1}^{*} u_{2}^{*} \cdots u_{k}^{*}$.

For any word $w \in \Sigma^{+}$, the primitive root of $w$ is denoted by $\rho(w)$ and corresponds to the minimal word such that $w \in(\rho(w))^{*}$.

\subsection{Poly-slender context free languages}

We review the definitions and results presented in [7] that will be fundamental ingredients throughout this paper.

Definition 1 (Dyck loop). Let $z=z_{1} z_{2} \cdots z_{2 k}$ be a Dyck word on $\{[]$,$\} of$ length $2 k$. Let $l_{i}$ and $r_{i}$ denote the respective positions of the $i$-th opening parenthesis [ and its corresponding closing one] in $z$. Given some words $y_{0}, \cdots, y_{2 k}$, $x_{1}, \cdots, x_{2 k}$, consider the map $h_{n_{1}, \cdots, n_{k}}\left(z_{1} z_{2} \cdots z_{2 k}\right)=y_{0} x_{1}^{e_{1}} y_{1} x_{2}^{e_{2}} y_{2} \cdots x_{2 k}^{e_{2 k}} y_{2 k}$ where each pair of parentheses shares the same exponent: $e_{l_{i}}=e_{r_{i}}=n_{i}$.

$A k$-Dyck loop is any set $D=\left\{h_{n_{1}, \cdots, n_{k}}\left(z_{1} z_{2} \cdots z_{2 k}\right): n_{i} \geq 0\right\}$ for some underlying Dyck word $z_{1} z_{2} \cdots z_{2 k}$ and words $x_{i}, y_{i}$.

Example 1. $\left\{a b^{n_{1}} a(b a)^{n_{2}+1} a^{n_{2}+1}(b a)^{n_{1}+n_{3}+2} b^{n_{3}}: n_{1}, n_{2}, n_{3} \geq 0\right\}$ is a 3-Dyck loop for the underlying Dyck word [[]][] and words $y_{0}=a, x_{1}=b, y_{1}=a b a$, $x_{2}=b a, y_{2}=a, x_{3}=a, y_{3}=\varepsilon, x_{4}=b a, y_{4}=b a b, x_{5}=a b, y_{5}=a, x_{6}=b, y_{6}=\varepsilon$.

Example 2. $\left\{a^{n_{1}} b^{n_{1}+n_{2}} a^{n_{2}}: n_{1}, n_{2} \geq 0\right\}$ is a 2-Dyck loop for the underlying Dyck word [][] and words $x_{1}=x_{4}=a, x_{2}=x_{3}=b, y_{0}=y_{1}=y_{2}=y_{4}=\varepsilon$.

The structure of poly-slender CFL finely corresponds to Dyck loops:

Theorem 1. For any $k \geq 0$, a context-free language is $k$-poly-slender if and only if it is a finite union of $(k+1)$-Dyck loops.

The following notion captures whether the position of some word $w$ can be distinguished or not.

Definition 2 (Link). Let $u, v \in \Sigma^{+}$and $w \in \Sigma^{*}$. The word $w$ links $u$ with $v$ $(\operatorname{link}(u, w, v))$ if and only if $\rho(u) w=w \rho(v)$. That means $\rho(u)=p q, \rho(v)=q p$ and $w=(p q)^{*} p$ for some $p, q$. And so $u^{m} w v^{n}$ is a prefix of $(p q)^{*}$ 
Example 3. With $x_{i}$ and $y_{i}$ as defined in Example 1, $\operatorname{link}\left(x_{4}, y_{4}, x_{5}\right)$ holds but $\operatorname{link}\left(x_{i}, y_{i}, x_{i+1}\right)$ does not hold for $i=1,2,3,5$.

With $x_{i}$ and $y_{i}$ as defined in Example 2, $\operatorname{link}\left(x_{2}, y_{2}, x_{3}\right)$ holds but neither $\operatorname{link}\left(x_{1}, y_{1}, x_{2}\right)$ nor $\operatorname{link}\left(x_{3}, y_{3}, x_{4}\right)$ holds.

We will make great use of the following lemma:

Lemma 1. Consider some words $x_{i} \in \Sigma^{+}$and $y_{i} \in \Sigma^{*}$, and some non-negative integers $n_{i}, m_{i}$. Denote $w=y_{0} x_{1}^{n_{1}} y_{1} x_{2}^{n_{2}} y_{2} \cdots x_{r}^{n_{r}} y_{r}, w^{\prime}=y_{0} x_{1}^{m_{1}} y_{1} x_{2}^{m_{2}} y_{2} \cdots x_{r}^{m_{r}} y_{r}$ and assume that $\operatorname{link}\left(x_{i}, y_{i}, x_{i+1}\right)$ holds for no $i$.

Then there is a constant $N_{0}$, depending only on the lengths of the words $x_{i}$ and $y_{i}$, such that, if $n_{i}, m_{i}$ are larger than $N_{0}$ and there is $i$ with $n_{i} \neq m_{i}$, then $w \neq w^{\prime}$.

For a general overview, we recall the result of Latteux and Thierrin [8] and the one of Ginsburg and Spanier [6]:

Theorem 2. A context-free language is poly-slender if and only if it is bounded.

Theorem 3. The family of bounded languages is the smallest family which contains all finite languages and is closed under the following operations:

1. union

2. catenation

3. $(x, y)^{\star} L=\bigcup_{n \geq 0} x^{n} L y^{n}$ for any $x, y$ words

\subsection{Real time one-way cellular automaton}

A one-way cellular automaton is a one-dimensional array of finite automata (the cells) indexed by $\mathbb{N}$. The cells evolve synchronously at discrete time steps. Each cell takes one value from a finite set of states $Q$. At each step, the evolution of a cell is defined by its own state and the state of its right neighbor according to a transition function $\delta$. Formally, denoting $\langle c, t\rangle$ the state of the cell $c$ at time $t$, $\langle c, t+1\rangle=\delta(\langle c, t\rangle,\langle c+1, t\rangle)$.

In order that an OCA acts as a language recognizer, we specify two subsets of $Q$ : the alphabet $\Sigma$ of input symbols and the set of accepting states $Q_{\text {accept }}$. The input mode is parallel. At initial time 0 , the $i$-th bit of the input word $w \in \Sigma^{*}$ is fed to the cell $i:\langle i, 0\rangle=w_{i}$. An OCA is said to accept (resp. reject) a word $w$ in real time, if on input $w$ the cell 0 enters an accepting (resp. non-accepting) state at time $|w|-1$. It corresponds to the minimal time for the cell 0 to know the whole input. A language is a real time OCA language if there exists some OCA $\left(Q, \Sigma, Q_{\text {accept }}, \delta\right)$ which accepts in real time exactly the words $w \in L$.

The computation of an OCA is usually represented by a time-space diagram (see Fig 1 ). The $t$-row corresponds to the cellular array at time $t$. Only those sites involved in the real time computation are depicted. As a matter of fact, there are two topologically equivalent ways to display the time-space diagram. We will use here the bilateral symmetric layout, i.e., the right one. 

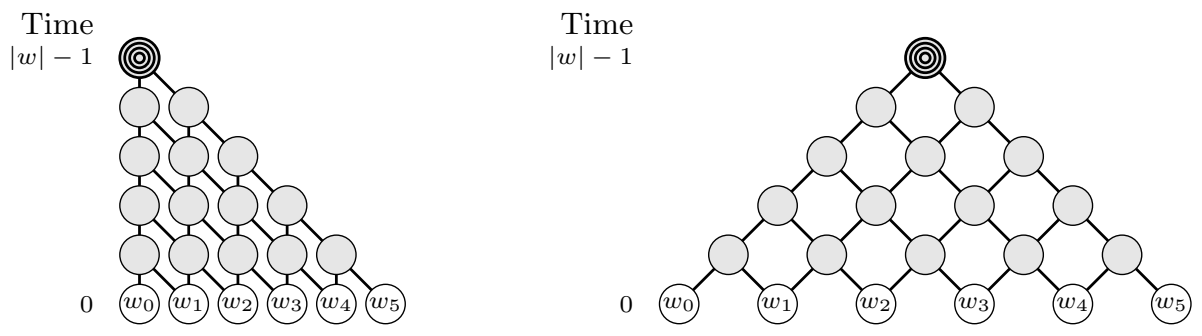

Fig. 1. Time-space diagram of a real time OCA

The real time OCA class is robust: introducing the notion of conjunctive grammar, Okhotin has exhibited a characterization of real time OCA in terms of a generating device [10]. Let us recall its statement, it will be one of the main ingredients in this paper. An alternating grammar is a grammar enhanced with a conjunctive operation symbolized by \&. Denoting $N$ the set of variables and $\Sigma$ the set of terminals, each production is of the form $A \rightarrow \alpha_{1} \& \cdots \& \alpha_{k}$ where $A \in N$ and $\alpha_{1}, \cdots, \alpha_{k} \in(A \cup \Sigma)^{*}$. Such a production denotes that the language generated by $A$ is the intersection of the languages generated by $\alpha_{1}, \cdots, \alpha_{k}$. Analogously to linear context free grammars, a linear conjunctive grammar is defined as an alternating grammar with the restriction that for every production $A \rightarrow \alpha_{1} \& \cdots \& \alpha_{k}$, no $\alpha_{i}$ has more than one instance of a variable. An algorithm describing how to recognize any linear CFL on a real time OCA was already known [3]. More radically, to extend linear context free grammars with the conjunctive operation \& leads to a complete characterization of real time OCA [10]:

Theorem 4. A language $L$ is recognized in real time by an $O C A$ if and only if $L$ is generated by a linear conjunctive grammar.

Let us recall the conversion from a real time OCA to a linear conjunctive grammar that we will need later. See Fig. 2 to get some insight about the translation.

Lemma 2. Given any language $L$ recognizable by some real time OCA $\mathcal{A}=\left(Q, \Sigma, Q_{\text {accept }}, \delta\right), L$ is generated by the linear conjunctive grammar $\mathcal{G}=\left(\Sigma,\{S\} \cup\left\{A_{q}: q \in Q\right\}, S, \mathcal{R}\right)$ where $\mathcal{R}$ contains the following rules:

$$
\begin{aligned}
& S \rightarrow A_{q} \text { for all } q \in Q_{\text {accept }} \\
& A_{a} \rightarrow a \text { for all } a \in \Sigma \\
& A_{\delta(g, d)} \rightarrow A_{g} b \& c A_{d} \text { for all } g, d \in Q \text { and all } b, c \in \Sigma
\end{aligned}
$$

At last, let us give some examples and properties of real time OCA to illustrate their abilities and limits. The Dyck language, the linear-slender CFL $\left\{a^{n} b^{n+m} a^{m}: n, m \geq 0\right\}$, the inherently exponentially ambiguous CFL $L^{*}$ with $L=\left\{a^{i} b^{j} c^{k}: i=j\right.$ or $\left.j=k\right\}$ [9], the non CFL languages $\left\{a^{n} b^{n} c^{n}: n \geq 0\right\}$ and $\left\{a^{n} b^{2^{n}}: n \geq 0\right\}$ with a non semilinear Parikh image, are all real time OCA languages. In addition, real time OCA languages include all linear CFL and visible 


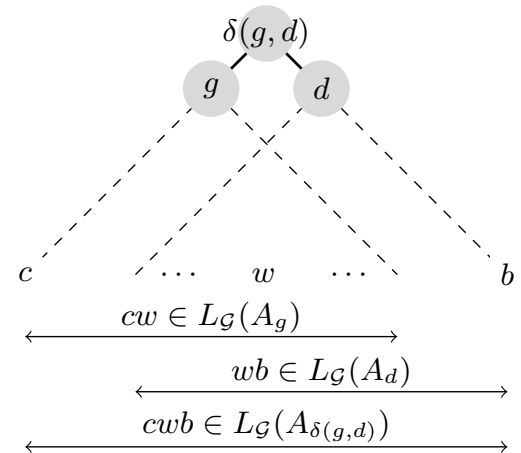

Fig. 2. The OCA's transition $\delta(g, d)$ is converted into the rule $A_{\delta(g, d)} \rightarrow A_{g} b \& c A_{d}$

pushdown languages $[3,11]$. On the other hand, several languages are known not to be real time OCA: all non regular languages over a one letter alphabet, the inherently ambiguous CFL $L_{1} L_{1}$ square of the linear CFL $L_{1}=\left\{1^{k} 0 u 10^{k}\right.$ : $\left.k>0, u \in\{0,1\}^{*}\right\}$, the language $\left\{u v u: u, v \in\{0,1\}^{*},|u|>1\right\}$, the deterministic CFL (to be more precise: LL(1) language) $\left\{c^{m} a^{l_{0}} b a^{l_{1}} b \cdots a^{l_{m}} b \cdots a^{l_{z}} b d^{n}\right.$ : $\left.m, n, l_{i} \geq 0, z \geq 1, l_{m}=n\right\}[1,12,13,11]$.

Further, the real time OCA class is closed under boolean operations, reverse, * operation (the one defined in Theorem 3), left and right concatenation with regular languages. The proofs are folklore. A contrario, the real time OCA class is not closed under morphism, concatenation, Kleene star and cycle $[4,12,14]$.

\subsection{Poly-slender context free languages and real time one-way cellular automata}

The question behind this paper is whether the poly-slender CFL are real time OCA languages. According to Theorem 3, the poly-slender CFL are the smallest family which contains all finite languages and is closed under union, concatenation and $\star$ operation. Of course, real time OCA languages include all finite languages and are closed under union and $\star$ operation. This is easy to prove using the grammar characterization of real time OCA. The problematic point is concatenation: can we assert that the concatenation of two Dyck loops is a real time OCA language? We do not answer this question but in the simple case: 1-Dyck loops. Precisely we will show the following result:

Theorem 5. Linear-slender context-free languages are recognizable in real time by one-way cellular automata.

The rest of the paper will be devoted to the proof of this theorem. Concretely we have to show that 2-Dyck loops are recognized in real time by OCA. 


\section{Recognition of 2-Dyck loops by real time OCA}

As an introduction, we may observe that 1-Dyck loops are real time OCA languages. Indeed a 1-Dyck loop corresponds to a set $D=\left\{y_{0} x_{1}^{n} y_{1} x_{2}^{n} y_{2}: n \in \mathbb{N}\right\}$ for some words $y_{0}, x_{1}, y_{1}, x_{2}, y_{2}$. It is a linear CFL and so it is a real time OCA language. As a consequence, all constant-slender languages are real time OCA languages.

Let us now consider 2-Dyck loops. According to whether the underlying Dyck word is [[]] or [][], they are of shape $\left\{y_{0} x_{1}^{n_{1}} y_{1} x_{2}^{n_{2}} y_{2} x_{3}^{n_{2}} y_{3} x_{4}^{n_{1}} y_{4}: n_{1}, n_{2} \geq 0\right\}$ or $\left\{y_{0} x_{1}^{n_{1}} y_{1} x_{2}^{n_{1}} y_{2} x_{3}^{n_{2}} y_{3} x_{4}^{n_{2}} y_{4}: n_{1}, n_{2} \geq 0\right\}$ A first simplification is to assume that the two ends $y_{0}$ and $y_{4}$ are empty knowing that if $L$ is a real time OCA language then so is $\left\{y_{0}\right\} L\left\{y_{4}\right\}$ whatever the words $y_{0}$ and $y_{4}$ are. We will suppose also that the words $x_{i}$ are non-empty, the degenerate cases being easy to handle.

\subsection{The underlying Dyck word is [[]].}

That is the simple case. The corresponding Dyck loop $\left\{x_{1}^{n_{1}} y_{1} x_{2}^{n_{2}} y_{2} x_{3}^{n_{2}} y_{3} x_{4}^{n_{1}}\right.$ : $\left.n_{1}, n_{2} \geq 0\right\}$ is clearly a linear CFL and so it is real time OCA recognizable. Here it is basically the closure under the $\star$ operation which is involved.

\subsection{The underlying Dyck word is [][].}

Now it is the closure under concatenation of 1-Dyck loops which is involved.

Case 1. $\operatorname{link}\left(x_{i}, y_{i}, x_{i+1}\right)$ holds for no $i=1,2,3$

Let $N_{0}$ be a constant as defined in Lemma 1 . The Dyck loop can be divided into three subsets $D_{1}, D_{2}$ and $D_{3}$, where

$$
\begin{aligned}
& D_{1}=\left\{x_{1}^{n_{1}} y_{1} x_{2}^{n_{1}} y_{2} x_{3}^{n_{3}} y_{3} x_{4}^{n_{3}}: n_{1}<N_{0}, n_{3} \in \mathbb{N}\right\}, \\
& D_{2}=\left\{x_{1}^{n_{1}} y_{1} x_{2}^{n_{1}} y_{2} x_{3}^{n_{3}} y_{3} x_{4}^{n_{3}}: n_{1} \in \mathbb{N}, n_{3}<N_{0}\right\}, \\
& D_{3}=\left\{x_{1}^{n_{1}} y_{1} x_{2}^{n_{1}} y_{2} x_{3}^{n_{3}} y_{3} x_{4}^{n_{3}}: n_{1}, n_{3} \geq N_{0}\right\} .
\end{aligned}
$$

Observe that $D_{1}=\bigcup_{0 \leq i<N_{0}}\left\{x_{1}^{i} y_{1} x_{2}^{i} y_{2} x_{3}^{n} y_{3} x_{4}^{n}: n \in \mathbb{N}\right\}$ is a finite union of linear CFL's and thus is alinear CFL. The subset $D_{2}$ is as well a linear CFL. Further, Lemma 1 ensures that $D_{3}$ can be specified as the intersection of two linear CFL's : $D_{3}=\left\{x_{1}^{n_{1}} y_{1} x_{2}^{n_{1}} y_{2} x_{3}^{n_{3}} y_{3} x_{4}^{n_{4}}: n_{1}, n_{3}, n_{4} \geq N_{0}\right\} \cap\left\{x_{1}^{n_{1}} y_{1} x_{2}^{n_{2}} y_{2} x_{3}^{n_{3}} y_{3} x_{4}^{n_{3}}\right.$ : $\left.n_{1}, n_{2}, n_{3} \geq N_{0}\right\}$ and so is real time recognizable by an OCA.

Case 2. $\operatorname{link}\left(x_{i}, y_{i}, x_{i+1}\right)$ holds for one $i=1,2,3$

The case where $y_{1}$ links $x_{1}$ with $x_{2}$ (or in a symmetric way $y_{3}$ links $x_{3}$ with $x_{4}$ ) does not present any difficulty. By Definition 2, $y_{1}$ links $x_{1}$ with $x_{2}$ if $\rho\left(x_{1}\right)=p q$, $\rho\left(x_{2}\right)=q p$ and $y_{1}=(p q)^{\gamma} p$ for some words $p, q$ and integer $\gamma$. Setting $x_{1}=(p q)^{\alpha}$, $x_{2}=(q p)^{\beta}, x_{1}^{n_{1}} y_{1} x_{2}^{n_{2}} y_{2}$ can be rewritten as $(p q)^{(\alpha+\beta) n_{1}+\gamma} p y_{2}$ and thus specifies a regular language. Moreover $x_{3}^{n_{3}} y_{3} x_{4}^{n_{4}}=x_{3}^{n_{3}} y_{3} x_{4}^{n_{3}}$ corresponds to a linear CFL. Their concatenation is linear CFL and so real time recognizable by an OCA. 
It remains to examine the most technical case where $y_{2}$ links $x_{2}$ with $x_{3}$ but $\operatorname{link}\left(x_{i}, y_{i}, x_{i+1}\right)$ does not hold for $i=1$ and $i=3$. As $y_{2}$ links $x_{2}$ with $x_{3}$ there exists some $p, q$ and $\alpha, \beta, \gamma$ such that $x_{2}=(p q)^{\alpha}, x_{3}=(q p)^{\beta}$ and $y_{2}=(p q)^{\gamma} p$. Then $x_{1}^{n_{1}} y_{1} x_{2}^{n_{2}} y_{2} x_{3}^{n_{3}} y_{3} x_{4}^{n_{4}}$ can be rewritten as $x_{1}^{n_{1}} y_{1}(p q)^{\alpha n_{1}+\beta n_{3}+\gamma} p y_{3} x_{4}^{n_{3}}$. Setting $z_{1}=x_{1}, z_{2}=y_{1}, z_{3}=p q, z_{4}=p y_{3}, z_{5}=x_{4}$, such 2-Dyck loops can be reshaped into $z_{1}^{n_{1}} z_{2} z_{3}^{\alpha n_{1}+\beta n_{3}+\gamma} z_{4} z_{5}^{n_{3}}$ with the properties that $z_{2}$ does not link $z_{1}$ with $z_{3}$ and, readily verifiable, $z_{4}$ does not link $z_{3}$ with $z_{5}$. So our task is to show that $\left\{z_{1}^{n} z_{2} z_{3}^{\alpha n+\beta m+\gamma} z_{4} z_{5}^{m}: n, m \geq 0\right\}$ is a real time OCA language. It will be done in two steps:

1. Given a five letters alphabet $\mathcal{A}=\left\{a_{1}, \cdots, a_{5}\right\}$, whatever $\alpha, \beta \geq 1$ and $\gamma \geq 0$ are, we will present a real time OCA which recognizes the language $L_{\alpha, \beta, \gamma}=\left\{a_{1}^{n} a_{2} a_{3}^{\alpha n+\beta m+\gamma} a_{4} a_{5}^{m}: n, m \geq 0\right\}$.

2. For any homomorphism $h$ on $\mathcal{A}$ such that neither $\operatorname{link}\left(h\left(a_{1}\right), h\left(a_{2}\right), h\left(a_{3}\right)\right)$ nor $\operatorname{link}\left(h\left(a_{3}\right), h\left(a_{4}\right), h\left(a_{5}\right)\right)$ holds, we will verify that $h\left(L_{\alpha, \beta, \gamma}\right)$ is a real time OCA language.

Proposition 1. The language $L_{\alpha, \beta, \gamma}=\left\{a_{1}^{n} a_{2} a_{3}^{\alpha n+\beta m+\gamma} a_{4} a_{5}^{m}: n, m>0\right\}$, where the symbols $a_{1}, \cdots, a_{5}$ are distinct, is recognizable in real time by an OCA.

Proof. The main ingredient is Culík's OCA which recognizes in real time the language $\left\{a^{n} b^{n+m} a^{m}: n, m \geq 0\right\}$ [2]. His construction makes ingenious use of a firing squad synchronization.

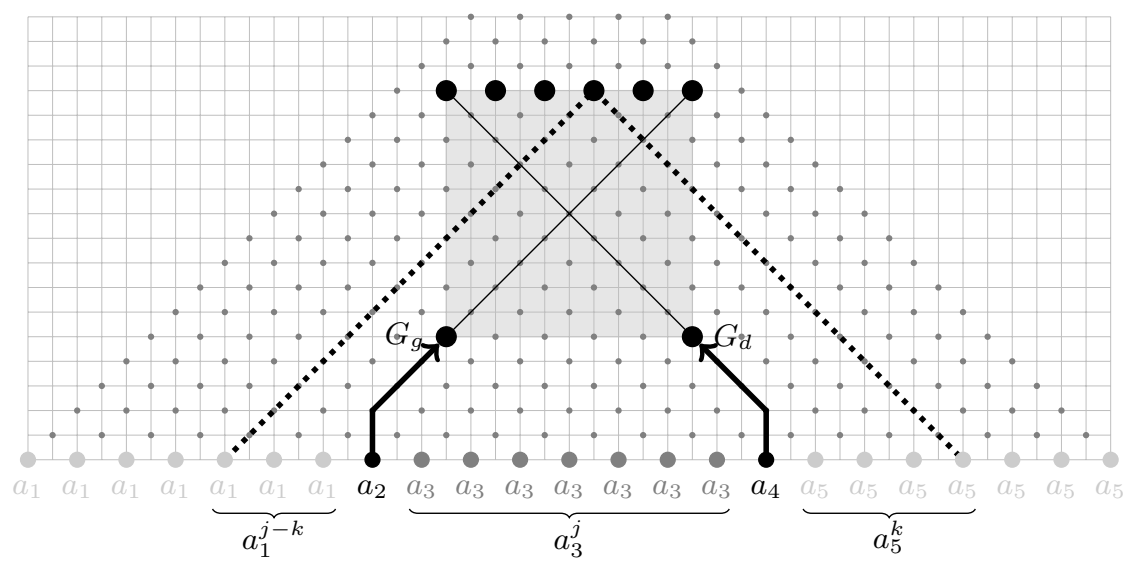

Fig. 3. Čulík's OCA

Let us begin by recalling the process (see Fig. 3). For convenience, we identify the sites of the OCA with integer coordinates $(x, y)$ where $x+y$ is even. In this way, the input symbols are placed at positions $(2 x, 0)$. The unique symbol $a_{2}$ is chosen to be at the origin $(0,0)$ and, when the input is of shape $a_{1}^{+} a_{2} a_{3}^{j} a_{4} a_{5}^{+}$, 
the unique symbol $a_{4}$ is at $(2 j+2,0)$. The process is set up using a firing squad synchronization with two generals $G_{g}$ and $G_{d}$ located according to the unique symbols $a_{2}$ and $a_{4}$. Precisely, the left general $G_{g}$ is at $(3,5)$ and the right general $G_{d}$ at $(2 j-1,5)$. Thus the synchronization occurs at points $(1+2 k, 1+2 j)$ for all $k$ with $0<k<j$. We check that the $k$-th firing points $(1+2 k, 1+2 j)$ corresponds to the output of the input subword which begins at $(2(k-j), 0)$ and ends at $(2(1+j+k), 0)$, namely the subword $a_{1}^{j-k} a_{2} a_{3}^{j} a_{4} a_{5}^{k}$.

Next, Culík showed that the construction can be adapted to recognize the languages $\left\{a^{i} b^{j} a^{k}: i, k \geq 0, m j+c=i+k\right\}$ for all $m \geq 1, c \geq 0$. Actually, the same approach works in these more general settings: it exists a real time OCA deciding the language $L_{\alpha, \beta, \gamma}=\left\{a_{1}^{i} a_{2} a_{3}^{j} a_{4} a_{5}^{k}: i, j, k \in \mathbb{N}, j=\alpha i+\beta k+\gamma\right\}$ for every non-negative rational numbers $\alpha, \beta, \gamma$. Let us outline the modified construction. Be warned that we will use rational coordinates but the technique to revert to an OCA diagram is standard. Firstly observe that we may reduce the range of the synchronization in locating the two generals later: with the left general $G_{g}$ at $(3+\gamma, 5+\gamma)$ and the right general $G_{d}$ at $(2 j-1-\gamma, 5+\gamma)$, the set of firing points becomes $\{(1+2 k+\gamma, 1+2 j-\gamma): k \in \mathbb{N}, 0<k<j-\gamma\}$. We explain now the construction in terms of geometric transformations. It will be achieved by the composition of two directional scalings (see Fig. 4).

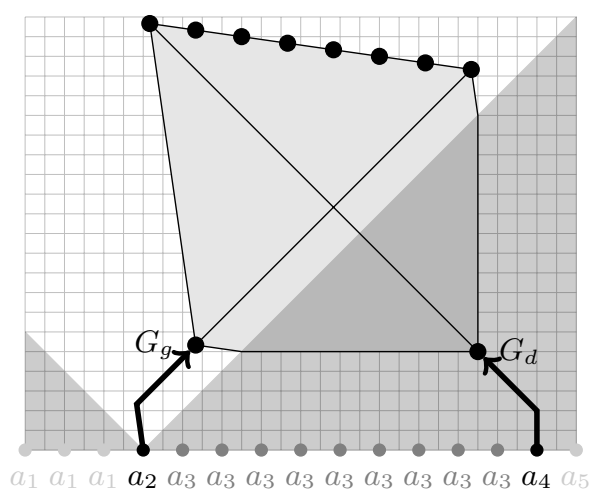

(a) Scaling in the up-left to down-right direction with factor $1 / \alpha=4 / 3$

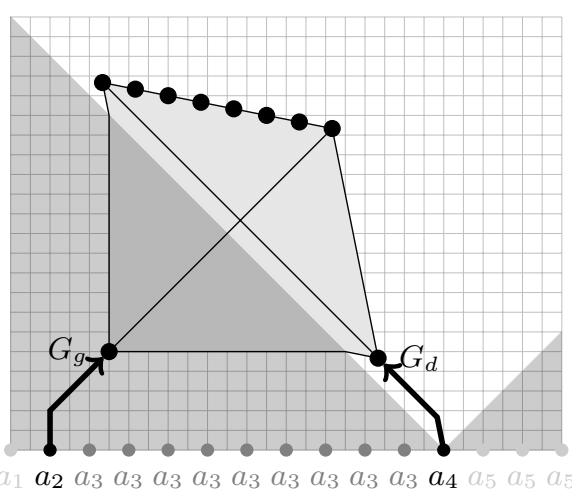

(b) Scaling in the up-right to down-left direction with factor $1 / \beta=2 / 3$

Fig. 4. Two scalings

Initiated from the unique symbol $a_{2}$, the first transformation starts at point $(0,0)$ and applies on its cone of consequences $\{(c, t):|c| \leq t\}$, i.e., the future light cone of $(0,0)$ which encompasses the set of points impacted by $(0,0)$. It leaves the line $t=c$ stable and scales by the factor $1 / \alpha$ in the up-left to downright direction according to the map $M_{g}=\left(\begin{array}{l}(1+\alpha) /(2 \alpha)(\alpha-1) /(2 \alpha) \\ (\alpha-1) /(2 \alpha)(1+\alpha) /(2 \alpha)\end{array}\right)$. Observe that the transformation is workable. Inside the cone, the neighborhood constraints 
are satisfied. The right side of the cone is stable. And the computation on the subword $a_{1}^{+}$occurring below the left side can easily be scaled.

The second transformation is symmetric. Initiated from the unique symbol $a_{4}$, it starts at point $(2 j+2,0)$ and applies on its cone of consequences $\{(c, t):|c-2 j-2| \leq t\}$. It leaves the line $t+c=2 j+2$ stable and scales by the factor $1 / \beta$ in the up-right to down-left direction according to the map

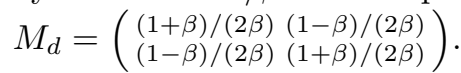

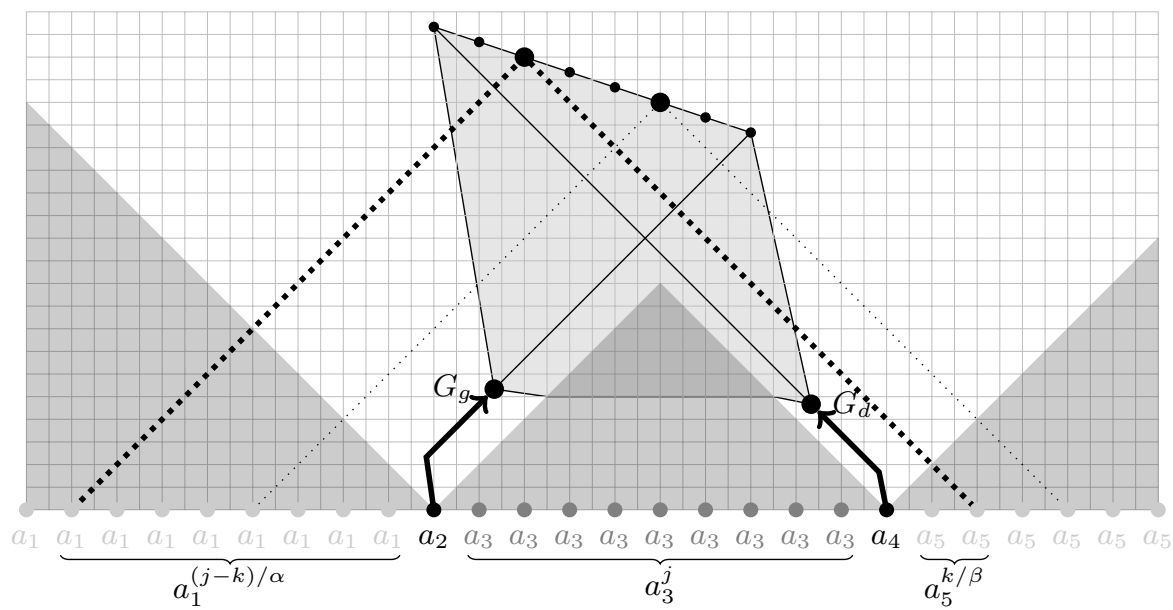

Fig. 5. Composition of two scalings

Now, the composition of these two scalings applies inside the cone of consequences of $(j+1, j+1):\{c, t):|c-j-1| \leq t-j-1\}$, intersection of the two cones issued from $a_{2}$ and $a_{4}$. See Fig. 5. It corresponds to the affine transformation with origin $(j+1, j+1)$ and matrix $M=M_{g} \times M_{d}=\left(\begin{array}{c}(\alpha+\beta) /(2 \alpha \beta)(\alpha-\beta) /(2 \alpha \beta) \\ (\alpha-\beta) /(2 \alpha \beta)(\alpha+\beta) /(2 \alpha \beta)\end{array}\right)$. Thus the firing points $\{(1+2 k+\gamma, 1+2 j-\gamma): k \in \mathbb{N}, 0<k<j-\gamma\}$ being inside the cone of $(j+1, j+1)$ are mapped to the points $\{(1+j-(j-k-\gamma) / \alpha+k / \beta$, $1+j+(j-k-\gamma) / \alpha+k / \beta): k \in \mathbb{N}, 0<k<j-\gamma\}$. Among these points, some of them match OCA's sites providing $k / \beta$ and $(j-k-\gamma) / \alpha$ are integers. Moreover such a point, with $(j-k-\gamma) / \alpha, k / \beta \in \mathbb{N}$, corresponds to the output of the input subword that begins at $(2(j-k-\gamma) / \alpha, 0)$ and ends at $(2 j+2+2 k / \beta, 0)$, i.e., the subword $a_{1}^{n} a_{2} a_{3}^{j} a_{4} a_{5}^{m}$ with $n=(j-k-\gamma) / \alpha, m=k / \beta$. To conclude just note that $\alpha n+\beta m+\gamma=j$.

Proposition 2. For any homomorphism $h$ on $\mathcal{A}$ such that neither $\operatorname{link}\left(h\left(a_{1}\right), h\left(a_{2}\right), h\left(a_{3}\right)\right)$ nor $\operatorname{link}\left(h\left(a_{3}\right), h\left(a_{4}\right), h\left(a_{5}\right)\right)$ holds, $h\left(L_{\alpha, \beta, \gamma}\right)$ is a real time OCA language. 
Proof. One has to be careful because real time OCA is not closed under morphism: each recursively enumerable language can be written as the image of a real time OCA language by a morphism [4]. But here we play with the image of languages with very simple structure.

First observe that $h\left(\left\{a_{1}^{n} a_{2} a_{3}^{\alpha n+\beta m+\gamma} a_{4} a_{5}^{m}: n<N_{0}\right.\right.$ or $\left.\left.m<N_{0}\right\}\right)$ is a linear $\mathrm{CFL}$, so to show that $h\left(\left\{a_{1}^{n} a_{2} a_{3}^{\alpha n+\beta m+\gamma} a_{4} a_{5}^{m}: n, m \geq N_{0}\right\}\right)$ is a real time OCA language will suffice. According to Proposition 1, there exists a real time OCA $\left(Q, \mathcal{A}, Q_{\text {accept }}, \delta\right)$ which recognizes the language $L=\left\{a_{1}^{n} a_{2} a_{3}^{\alpha n+\beta m+\gamma} a_{4} a_{5}^{m}\right.$ : $\left.n, m \geq N_{0}\right\}$. Following Okhotin [10], $L$ is defined by the linear conjunctive grammar $\mathcal{G}=\left(\mathcal{A},\{S\} \cup\left\{A_{q}: q \in Q\right\}, S, \mathcal{R}\right)$ where $\mathcal{R}$ contains the following rules:

$$
\begin{aligned}
& S \rightarrow A_{q} \text { for all } q \in Q_{a c c e p t} \\
& A_{a} \rightarrow a \text { for all } a \in \mathcal{A} \\
& A_{\delta(g, d)} \rightarrow A_{g} b \& c A_{d} \text { for all } g, d \in Q \text { and all } b, c \in \mathcal{A}
\end{aligned}
$$

Now let us make explicit, in the grammar rules, the bounded feature of the language. All words are of shape $a_{1}^{+} a_{2} a_{3}^{+} a_{4} a_{5}^{+}$. We denote by Follow $(i)$ the set of $j$ such that $a_{j}$ follows immediately $a_{i}$ in the expression $a_{1}^{+} a_{2} a_{3}^{+} a_{4} a_{5}^{+}$: Follow $(1)=\{1,2\}$, Follow $(2)=\{3\}$, Follow $(3)=\{3,4\}$, Follow $(4)=\{5\}$, Follow $(5)=\{5\}$. Then we rewrite $\mathcal{G}$ to $\mathcal{G}^{\prime}=\left(\mathcal{A},\{S\} \cup\left\{\left(A_{q}, 1,5\right): q \in Q_{\text {accept }}\right\} \cup\right.$ $\left.\left\{\left(A_{q}, i, j\right): q \in Q \backslash Q_{\text {accept }}, 1 \leq i \leq j \leq 5\right\}, S, \mathcal{R}^{\prime}\right)$ where $\mathcal{R}^{\prime}$ contains the following rules:

$$
\begin{aligned}
& S \rightarrow\left(A_{q}, 1,5\right) \text { for all } q \in Q_{a c c e p t} \\
& \left(A_{a_{i}}, i, i\right) \rightarrow a_{i} \text { for all } i=1, \cdots, 5 \\
& \left(A_{\delta(g, d)}, i, j\right) \rightarrow\left(A_{g}, i, s\right) a_{j} \& a_{i}\left(A_{d}, r, j\right) \text { for all } g, d \in Q \text { and all } i, j, r, s \\
& \quad \text { with } 1 \leq i \leq r \leq s \leq j \leq 5, r \in \text { Follow }(i), j \in \text { Follow }(s)
\end{aligned}
$$

To gain a better understanding of the last derivation rule, see Fig. 6 , it depicts the corresponding OCA transition. With such refinements, we get that $L_{\mathcal{G}^{\prime}}\left(A_{q}, i, j\right)$ is the subset of $L_{\mathcal{G}}\left(A_{q}\right)$ whose words begin with $a_{i}$, ends with $a_{j}$ and which are factors of $a_{1}^{+} a_{2} a_{3}^{+} a_{4} a_{5}^{+}$.

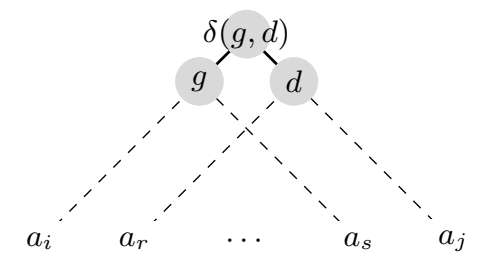

Fig. 6. Interpretation of the rule $\left(A_{\delta(g, d)}, i, j\right) \rightarrow\left(A_{g}, i, s\right) a_{j} \& a_{i}\left(A_{d}, r, j\right)$ with $i \leq r \leq$ $s \leq j, r \in$ Follow $(i), j \in$ Follow $(s)$

In addition, to meet later requirements, we replace all rules $\left(A_{a_{i}}, i, i\right) \rightarrow a_{i}$ with the rules $\left.\left(A_{\delta\left(a_{i_{1}}, \cdots, a_{i_{0}}\right.}\right), i_{1}, i_{N_{0}}\right) \rightarrow a_{i_{1}} \cdots a_{i_{N_{0}}}$ for all words $a_{i_{1}} \cdots a_{i_{N_{0}}}$ of 
length $N_{0}$ which are factors of $a_{1}^{N_{0}} a_{2} a_{3}^{N_{0}} a_{4} a_{5}^{N_{0}}$. It does not alter the expressiveness of the grammar.

Finally, in replacing each symbol $a_{i}$ with its image $h\left(a_{i}\right), \mathcal{G}$ is rewritten to $\mathcal{H}=\left(\Sigma,\{S\} \cup\left\{\left(A_{q}, i, j\right): q \in Q, 1 \leq i \leq j \leq 5\right\}, S, \mathcal{R}^{\prime \prime}\right)$ where $\mathcal{R}^{\prime \prime}$ contains the following rules:

$$
\begin{aligned}
& S \rightarrow\left(A_{q}, 1,5\right) \text { for all } q \in Q_{\text {accept }} \\
& \left.\left(A_{\delta\left(a_{i_{1}}, \cdots, a_{i_{0}}\right.}\right), i_{1}, i_{N_{0}}\right) \rightarrow h\left(a_{i_{1}} \cdots a_{i_{N_{0}}}\right) \text { for all words } a_{i_{1}} \cdots a_{i_{N_{0}}} \text { of } \\
& \text { length } N_{0} \text { which are factors of } a_{1}^{N_{0}} a_{2} a_{3}^{N_{0}} a_{4} a_{5}^{N_{0}} \\
& \left(A_{\delta(g, d)}, i, j\right) \rightarrow\left(A_{g}, i, s\right) h\left(a_{j}\right) \& h\left(a_{i}\right)\left(A_{d}, r, j\right) \text { for all } g, d \in Q \text { and all } \\
& i, j, r, s \text { with } 1 \leq i \leq r \leq s \leq j \leq 5, r \in \text { Follow }(i), j \in \text { Follow }(s)
\end{aligned}
$$

Clearly, $h\left(L_{\mathcal{G}}(S)\right) \subseteq L_{\mathcal{H}}(S)$. Let us ensure that they are equal and, more specifically, that $L_{\mathcal{H}}(A, i, j) \subseteq h\left(L_{\mathcal{G}}(A, i, j)\right)$ for every variable $(A, i, j)$. The proof is done by induction on the height of the parse trees. The inductive assumption is that all words generated within $\mathcal{H}$ by a tree of height $h$ and root node $(A, i, j)$ are images by $h$ of words generated within $\mathcal{G}$ by a tree of height $h$ and root node $(A, i, j)$.

The base case. The trees of height 1 within $\mathcal{H}$ display the derivations $\left(A_{q}, i_{1}, i_{N_{0}}\right) \rightarrow h\left(a_{i_{1}} \cdots a_{i_{N_{0}}}\right)$ whereas their counterparts within $\mathcal{G}$ display the derivations $\left(A_{q}, i_{1}, i_{N_{0}}\right) \rightarrow a_{i_{1}} \cdots a_{i_{N_{0}}}$.

The inductive step. We focus on the trees with root node $\left(A_{q}, 1,5\right)$ and omit the ones with root $\left(A_{q}, i, j\right)$ when $i>1$ or $j<5$ that are easier to handle. Given any tree $T$ within $\mathcal{H}$ of height $h+1>1$ and root $\left(A_{q}, 1,5\right)$. The root node expands into two subtrees of height at most $h$ according to some rule $\left(A_{q}, 1,5\right) \rightarrow$ $\left(A_{g}, 1, s\right) h\left(a_{5}\right) \& h\left(a_{1}\right)\left(A_{d}, r, 5\right)$ where $\delta(g, d)=q, s$ is 4 or 5 and $r$ is 1 or 2 . By assumption, $\left(A_{g}, 1, s\right)$ produces the image by $h$ of a word $a_{1}^{n_{1}} a_{2} a_{3}^{n_{2}} a_{4} a_{5}^{n_{3}}$ with $n_{1}>0, n_{2}>N_{0}, n_{3} \geq 0$ and $\left(A_{d}, r, 5\right)$ produces the image by $h$ of a word $a_{1}^{m_{1}} a_{2} a_{3}^{m_{2}} a_{4} a_{5}^{m_{3}}$ with $m_{1} \geq 0, m_{2}>N_{0}, m_{3}>0$. Hence the root node produces $h\left(a_{1}^{n_{1}} a_{2} a_{3}^{n_{2}} a_{4} a_{5}^{n_{3}+1}\right)=h\left(a_{1}^{m_{1}+1} a_{2} a_{3}^{m_{2}} a_{4} a_{5}^{m_{3}}\right)$. Next we may notice that, in the proof of Lemma 1, the hypothesis that the first exponents $n_{1}$ and $m_{1}$ are greater than $N_{0}$ is not used, as well, (in handling the word backward) for the last exponents $n_{r}$ and $m_{r}$. Bearing in mind that neither $\operatorname{link}\left(h\left(a_{1}\right), h\left(a_{2}\right), h\left(a_{3}\right)\right)$ nor $\operatorname{link}\left(h\left(a_{3}\right), h\left(a_{4}\right), h\left(a_{5}\right)\right)$ holds, all prerequisites to apply Lemma 1 are satisfied. So $n_{1}=m_{1}+1, n_{2}=m_{2}, n_{3}+1=m_{3}$. It follows that $a_{1}^{n_{1}} a_{2} a_{3}^{n_{2}} a_{4} a_{5}^{n_{3}+1}$ is indeed represented by a tree within $\mathcal{G}$ structured as $T$.

\section{Conclusion}

Based on the precise characterization of the poly-slender CFL in terms of Dyck loops given by Ilie, Rozenberg and Salomaa, we have shown that linear-slender CFL are real time OCA languages. More than the result in itself, the approach appears interesting. It mixes algorithmic constructions with grammar tools and combinatoric properties: Starting from a real time OCA recognizing a specific language, here a variant of Culík's one, we make use of Okhotin's result to 
translate it in terms of a linear conjunctive grammar. Then in modifying the grammar, we capture a wider set of real time OCA languages structured like the starting one.

Now the challenge would be to show that poly-slender CFL are also real time OCA languages, in other words, that all Dyck loops are real time OCA languages. For that purpose, a key step would be to exhibit a generalization of Culík's construction to handle languages of shape $a_{0} b_{1}^{n_{1}} a_{1} b_{2}^{n_{2}} a_{2} \cdots b_{r}^{n_{r}} a_{r}$ where the integers $n_{1}, \cdots, n_{r}$ are linear combinations of $k$ integers and the symbols $a_{i}$, $b_{i}$ are distinct.

\section{References}

1. Christian Choffrut and Karel Čulík II. On real-time cellular automata and trellis automata. Acta Informatica, 21(4):393-407, November 1984.

2. Karel Čulík II. Variations of the firing squad problem and applications. Information Processing Letters, 30(3):152 - 157, 1989.

3. Karel Culík II, Jozef Gruska, and Arto Salomaa. Systolic trellis automata. I, II. International Journal Computer Mathematics, 16:3-22, 1984.

4. Karel Čulík II, Jozef Gruska, and Arto Salomaa. Systolic trellis automata: Stability, decidability and complexity. Information and Control, 71(3):218-230, 1986.

5. Charles R. Dyer. One-way bounded cellular automata. Information and Control, 44(3):261-281, 1980.

6. Seymour Ginsburg and Edwin H. Spanier. Bounded algol-like languages. Transactions of the American Mathematical Society, 113:333-368, 1964.

7. Lucian Ilie, Grzegorz Rozenberg, and Arto Salomaa. A characterization of polyslender context-free languages. Theoretical Informatics and Applications, 34(1):77$86,2000$.

8. Michel Latteux and Gabriel Thierrin. On bounded context-free languages. Elektronische Informationsverarbeitung und Kybernetik, 20(1):3-8, 1984.

9. Mohamed Naji. Ambiguity of context-free languages as a function of the word length. FB Informatik, Goethe Universität, Frankfurt am Main, 1998.

10. Alexander Okhotin. On the equivalence of linear conjunctive grammars and trellis automata. RAIRO Informatique Théorique et Applications, 38(1):69-88, 2004.

11. Alexander Okhotin. Comparing linear conjunctive languages to subfamilies of the context-free languages. In SOFSEM 2011: Theory and Practice of Computer Science, Nový Smokovec, Slovakia, LNCS 6543, pages 431-443, 2011.

12. Véronique Terrier. On real time one-way cellular array. Theoretical Computer Science, 141(1-2):331-335, 1995.

13. Véronique Terrier. Language not recognizable in real time by one-way cellular automata. Theoretical Computer Science, 156(1-2):281-287, 1996.

14. Véronique Terrier. Closure properties of cellular automata. Theoretical Computer Science, 352(1-3):97-107, 2006. 